\title{
Fatores associados à depressão em idoso
}

\author{
Factors associated with depression in the elderly \\ Factores asociados a la depresión en ancianos
}

\begin{abstract}
Fabiana Pinheiro Ramos ${ }^{1}$, Silmara Correia da Silva ${ }^{1}$, Daniela Fernanda de Freitas ${ }^{2 \star}$, Lílian Mendes Borburema Gangussu², Audrey Handyara Bicalho², Berenilde Valéria de Oliveira Sousa ${ }^{2}$; Zuíla Maria de Jesus Rametta ${ }^{3}$, Flávia de Jesus Rametta ${ }^{4}$, Filipe de Jesus Rametta ${ }^{5}$, Letícia Prates Morais Rametta $^{6}$, Cláudio Ildeu Costa Nascimento ${ }^{7}$, Sérgio Henrique Sousa Santos ${ }^{2,8}$, Talita Antunes Guimarães ${ }^{1}$.
\end{abstract}

\section{RESUMO}

Objetivo: Compreender a depressão na terceira idade, o perfil e o processo de prevenção e tratamento dos sinais e sintomas. Metodologia: A revisão integrativa de literatura foi feita utilizando as bases: Biblioteca Virtual da Saúde (BVS): Sistema Latino Americano e do Caribe de informação em Ciências da Saúde (LILACS), Scientific Electronic Library Online (Scielo). Ao final da busca foram escolhidos os estudos científicos publicados nos últimos 10 anos, epublicados na íntegra, no período de 2007 a 2017. Foram utilizados os seguintes termos: "Depressão em idosos"; "Sintomas de Depressão"; "Prevenção da Depressão" e "Tratamento da Depressão". Resultados: Após confrontarmos os resultados encontrados com a revisão da literatura que a depressão no idoso é um dos transtornos psiquiátricos mais frequentes. Acredita-se que conforme o aumento da idade, mais sintomas depressivos são apresentados, através das queixas intensificadas de doenças e a presença do quadro de ansiedade. Para melhor qualidade o idoso necessita ter conhecimento sobre sua condição e as doenças que podem the acometer, o que a auxilia na promoção da própria saúde, reduzindo o sofrimento. Contribuindo para o envelhecimento ativo, tornando-a participativa na sociedade, onde demonstra autonomia sobre a própria velhice. Considerações finais: Evidenciou-se a partir da literatura que existe a necessidade de agir de modo preventivo, junto aos idosos, é premente. A prática e o desenvolvimento de atividades de lazer têm-se mostrado na vida dos idosos como um fator de grande importância. O lazer contribui para um melhor estado de espírito e no caso dos mais idosos, diminui os efeitos decorrentes do processo de envelhecimento.

Palavras chave: Depressão, Sintomas, Prevenção, Tratamento.

\section{ABSTRACT}

Objective: To understand depression in the elderly, the profile and the process of prevention and treatment of signs and symptoms. Methodology: Methodology: Integrative literature review was done using the bases: Virtual Health Library (VHL): Latin American and Caribbean System of information in Health Sciences (LILACS), Scientific Electronic Library Online (Scielo). At the end of the search, the scientific studies published in the last 10 years, published in full, between 2007 and 2017 were selected. The following terms were used:

\footnotetext{
${ }^{1}$ Faculdade de Saúde e Desenvolvimento Humano Santo Agostinho - FASA, Montes Claros-MG.

2 Programa de Pós-graduação Ciências da Saúde - Unimontes, Montes Claros, MG.

${ }^{3}$ Hospital Universitário Clemente de Farias - Unimontes, Montes Claros-MG.

${ }^{4}$ Residente em Ortopedia, Hospital Hugol, Goiânia-GO.

${ }^{5}$ Médico Especialista em Medicina do Trabalho e Especialização Médica, Universidade do Oeste Paulista, Tenente da

Polícia Militar Paracatu-MG.

${ }^{6}$ Faculdade Tecsoma, Paracatu-MG.

${ }^{7}$ Escola Técnica de Saúde do Centro de Educação Profissional e Tecnológica da Unimontes, Montes Claros-MG.

${ }^{8}$ Universidade Federal de Minas Gerais - Instituto de Ciência Agrárias, Montes Claros-MG

* E-mail: danielafernandadefreitas@gmail.com
} 
"Depression in the elderly"; "Symptoms of Depression"; "Prevention of Depression" and "Treatment of Depression". Results: After comparing the results found with the literature review that depression in the elderly is one of the most frequent psychiatric disorders. It is believed that as the age increases, more depressive symptoms are presented, through intensified complaints of illness and the presence of anxiety. For better quality the elderly need to know about their condition and the diseases that can affect him, which helps him to promote his own health, reducing suffering. Contributing to active aging, making it participatory in society, where it demonstrates autonomy over one's own old age. Final considerations: It has been evidenced from the literature that there is a need to act in a preventive way, together with the elderly, it is urgent. The practice and development of leisure activities have been shown in the lives of the elderly as a factor of great importance. Leisure contributes to a better state of mind and, in the case of the elderly, reduces the effects of the aging process.

Keywords: Depression, Symptoms, Prevention, Treatment.

\section{RESUMEN}

Objetivo: Comprender la depresión en la tercera edad, el perfil y el proceso de prevención y tratamiento de los signos y síntomas. Metodología: La revisión integrativa de literatura fue hecha utilizando las bases: Biblioteca Virtual de la Salud (BVS): Sistema Latinoamericano y del Caribe de información en Ciencias de la Salud (LILACS), Scientific Electronic Library Online (Scielo). Al final de la búsqueda se escogieron los estudios científicos publicados en los últimos 10 años, expuestos en su totalidad, en el período de 2007 a 2017. Se utilizaron los siguientes términos: "Depresión en ancianos"; "Síntomas de depresión"; "Prevención de la Depresión" y "Tratamiento de la Depresión". Resultados: Después de confrontar los resultados encontrados con la revisión de la literatura que la depresión en el anciano es uno de los trastornos psiquiátricos más frecuentes. Se cree que según el aumento de la edad, más síntomas depresivos se presentan, a través de las quejas intensificadas de enfermedades y la presencia del cuadro de ansiedad. Para una mejor calidad, el anciano necesita tener conocimiento sobre su condición y las enfermedades que pueden acometer, lo que le ayuda a promover la propia salud, reduciendo el sufrimiento. Contribuyendo al envejecimiento activo, haciéndola participativa en la sociedad, donde demuestra autonomía sobre la propia vejez. Consideraciones finales: Se evidenció a partir de la literatura que existe la necesidad de actuar de modo preventivo, junto a los ancianos, es apremiante. La práctica y el desarrollo de actividades de ocio se han mostrado en la vida de los ancianos como un factor de gran importancia. El ocio contribuye a un mejor estado de ánimo y en el caso de los más ancianos, disminuye los efectos derivados del proceso de envejecimiento.

Palabras clave: Depresión, Síntomas, Prevención, Tratamiento.

\section{INTRODUÇÃO}

O envelhecimento populacional é um fenômeno mundial, sendo atualmente mais expressivo e impactante nos países em desenvolvimento. No Brasil, o crescimento repentino da população idosa é resultado de variáveis demográficas, bem como, das alterações sociais e culturais ocorridas, sendo estes causa e consequência desse aumento. Segundo o Instituto Brasileiro de Geografia e Estatística (IBGE) em 40 anos, a população idosa vai triplicar no País e passará de 19,6 milhões (10\% da população brasileira), em 2010, para 66,5 milhões de pessoas, em 2050 (29,3\%). As estimativas são de que a mudança no perfil da população ocorrerá em 2030, quando o número absoluto e o porcentual de brasileiros com 60 anos ou mais de idade irão exceder o de crianças de 0 a 14 anos, ainda as projeções apontam que em 2050 atingirá 38 milhões de idosos com depressão, superando a proporção de jovens na população (LEAL, 2016; BRASIL, 2010).

A depressão pode ser desencadeada por fatores biológicos, sendo a genética um fator significativo no desenvolvimento de um quadro depressivo. Além disso, fatores psicológicos causam perda da autonomia e agravamento de quadros patológicos preexistentes no idoso, assim como os fatores sociais que interferem na capacidade funcional, do autocuidado e nas suas relações sociais (NÓBREGA et al., 2015).

No processo dessa enfermidade o sexo feminino apresenta uma maior vulnerabilidade devido às situações de conflitos familiares, relacionamentos rompidos bem como aos fatores biológicos genéticos e hormonais. Outros fatores existentes incluem faixa etária, restrições socioeconômicas, baixa escolaridade, atribuições de 
personalidade, distúrbios do sono, inadequações da moradia, déficit no suporte social, eventos de vida estressantes, quadro psiquiátrico prévio, declínio cognitivo, restrições funcionais e morbidades, sejam elas crônicas ou agudas (RAMOS et al., 2017).

É bastante reduzido o diagnóstico de depressão em idosos, estima-se que $50 \%$ dos idosos depressivos não são diagnosticados pelos profissionais de saúde que exercem atividade na atenção primária, devido os sintomas serem semelhantes ao processo natural do envelhecimento. Algumas desses sintomas são, queixas físicas com fadiga, sono, falta de apetite e indisposição que podem ser confundidos pelo desafio adaptativo do envelhecimento (SOUZA et al., 2017).

Apesar de haver disponibilidade de mais de oito classes de antidepressivos, com aproximadamente 22 substâncias ativas no mercado mundial para o tratamento farmacológico da depressão, somente 30 a 35\% dos pacientes depressivos respondem ao tratamento com psicofármacos. Para a eventual remissão, faz-se necessário, portanto, a utilização de outros métodos de tratamento associados ao medicamentoso (MORAES et al., 2007).

$\mathrm{Na}$ sociedade, o número de idosos com depressão é comum, recorrente e frequentemente subdiagnosticada e sub-tratada, principalmente ao nível dos cuidados de saúde primária. As consequências na saúde pública do sub-tratamento da depressão no idoso irão aumentar, dado o envelhecimento crescente da população (ALMEIDA e QUINTÃO, 2012).

Esta pesquisa justifica-se pela abordagem preventiva além de possuir um caráter de valorização da saúde coletiva, ressalvando fatores que podem desencadear ou mesmo predispor ao começo do estado depressivo, avaliando as possíveis causas da depressão. Atividades preventivas como atividade física regular têm sido eficiente no tratamento da depressão, como também a utilização de jogos de memória, passeios, discussões saudáveis, leitura e conversas com o objetivo de aumentar a autoestima (CISCA, 2017).

A atividade física pode ser considerada eficaz no tratamento da depressão. Podendo propiciar benefícios agudos e crônicos. São eles: melhora no condicionamento físico; diminuição da perda de massa óssea e muscular; aumento da força, coordenação e equilíbrio; redução da incapacidade funcional, da intensidade dos pensamentos negativos e das doenças físicas; e promoção da melhoria do bem-estar e do humor (MORAES et al., 2007).

A ajuda da família nesse momento é de fundamental relevância para alcançar resultados satisfatórios, pois nesse período da vida muitos idosos se sentem incapazes, e são desprezados e resignados tanto pela sociedade, quanto pela família. A doença deve ser compreendida e tratada, paciência é a chave para compreender melhor os idosos nessa situação, a interação e as conversas são fundamentais para que o idoso não se isole cada vez mais, o assunto merece atenção especial, pois se não tratado devidamente pode trazer danos à saúde física e mental, o acompanhamento médico é sempre importante, mas o apoio da família também é essencial (LIMA et al., 2016).

Considerando a relevância desta temática, esta pesquisa objetivou a realização de uma análise descritiva acerca de compreender a depressão na terceira idade, o perfil e o processo de prevenção e tratamento dos sinais e sintomas.

\section{METODOLOGIA}

Foi elaborada uma revisão integrativa da literatura, visto que a esta agrega e simplifica resultados de pesquisas sobre um determinado tema, de maneira sistemática e organizada, apoiando o conhecimento do tema em questão. 
Segundo Souza et al. (2010), as etapas do procedimento de preparação da revisão integrativa foram: construção da pergunta norteadora, estabelecimento dos objetivos da revisão e critérios de inclusão e exclusão dos artigos; definição das informações a serem removidas das pesquisas; seleção dos artigos na literatura; análise dos resultados; discussão dos achados e apresentação da revisão.

Para coordenar esta revisão, elaborou-se a questão norteadora: Como é o perfil da depressão na terceira idade? Para a seleção dos artigos utilizou-se acesso online as bases de dados da Biblioteca Virtual da Saúde (BVS): Sistema Latino Americano e do Caribe de informação em Ciências da Saúde (LILACS), Scientific Electronic Library Online (Scielo). Foram eleitos estudos científicos publicados nos últimos 10 anos, estudos na íntegra, publicados no período de 2007 a 2017, e que consideraram a problemática sendo exclusos da amostra estudos que não atendiam os critérios de inclusão pré-estabelecidos ou que não havia validade científica. Para a procura dos conteúdos e a descrição dos conceitos foram utilizados os seguintes termos: "Depressão em idosos"; "Sintomas de Depressão"; "Prevenção da Depressão" e "Tratamento da Depressão".

Como critérios de eliminação, foram determinados: publicações dobradas nas bases de dados, teses, dissertações, revisões de literatura, publicações na forma de cartas, resenhas, comentários, artigos de opinião, relatos de experiência, resumos de anais, dossiês, relatórios de gestão e editoriais, livros, capítulos de livros, documentos governamentais, boletins informativos.

A discussão foi promovida com a bibliografia direcionada pela revisão e dissertação de conteúdos, cujo intuito incide na verificação e na construção de evidências que ajude na compreensão da depressão na terceira idade, o perfil e o processo de prevenção e tratamento dos sinais e sintomas.

A seleção resultou em um total de 16 artigos, dois artigos do ano de 2007 (12,5\%), um artigo do ano de $2010(6,25 \%)$, dois artigos do ano de 2012 (12,5\%), um artigo do ano de 2014 (6,25\%), cinco artigos do ano de 2015 (31,25\%), três artigos do ano de 2016 (18,75\%)e dois artigos do ano de 2017(12,5\%).

\section{RESULTADOS E DISCUSSÃO}

A seleção final resultou em um total de 10 artigos, conforme descrito na Tabela 1.

Tabela 1: Seleção dos artigos incluídos na pesquisa.

\begin{tabular}{|c|c|c|c|}
\hline Autores & Tipo de estudo & Ano & Principais resultados \\
\hline SOARES et al & Artigo original & 2017 & $\begin{array}{l}\text { A pesquisa mostrou prevalência de } \\
\text { depressão no sexo feminino e na renda } \\
\text { familiar um valor menor que três salários } \\
\text { mínimos. }\end{array}$ \\
\hline SOUSA et al & Artigo original & 2017 & $\begin{array}{l}\text { Identificaram-se prevalência de depressão } \\
\text { em idosos quando feita análise multivariada } \\
\text { de regressão, as variáveis significativas para } \\
\text { maior prevalência de sintomas de depressão } \\
\text { foram pessoas do sexo feminino, divorciadas, } \\
\text { sem religião e com doença crônica. }\end{array}$ \\
\hline $\begin{array}{l}\text { de ANDRADE et } \\
\text { al }\end{array}$ & Artigo original & 2016 & $\begin{array}{l}\text { Do total dos } 306 \text { participantes, } 245 \text { idosos } \\
\text { nunca receberam o diagnóstico de } \\
\text { depressão, } 203 \text { conheceram alguém } \\
\text { acometido pela doença e } 130 \text { idosos } \\
\text { apresentaram conhecimento intermediário } \\
\text { em relação à depressão, caracterizando o } \\
\text { maior grupo. }\end{array}$ \\
\hline
\end{tabular}


LIMA et al Artigo original

GULLICH et al Artigo original

MIGUEL et al. Artigo original

2015

RACHEL et al Artigo original

2015

CANTÃO et al Artigo original

2015

RUFINE et al

Artigo original

2014

ALMEIDA

e Artigo original

QUINTÃO

2016

2016
A pesquisa mostrou a importância do estímulo ao autocuidado, ativação e engajamento deste público e familiares em atividades educativas, treinamento profissional de saúde e ampliação do sistema como fatores importantes ao cuidado destes usuários.

Os resultados obtidos neste estudo revelam que participar de atividades coletivas de lazer, como baile e culto religioso, ou realizar atividade física regular mostraram-se protetores à ocorrência de depressão. Revelam necessidade e a possibilidade de tratamento e manejo dessa doença em nível coletivo.

Pode-se concluir que o envelhecimento, dor crônica e depressão, ainda são poucos e até inconclusivos para ser a causa da depressão, o que demonstra a necessidade de aumento nas pesquisas a respeito da população que cresce tão rapidamente.

Identificaram-se associações significativas entre sintomas depressivos e viuvez, observando-se que os sintomas depressivos foram mais frequentes entre as mulheres, os de baixa escolaridade e os que não utilizaram serviços de saúde.

Observou-se que o álcool foi a droga mais utilizada entre os homens e o tabaco entre as mulheres, o episódio depressivo foi a situação de maior ocorrência entre os sexos.

Conclui-se que em todos os modelos propostos a qualidade de vida foi capaz de inibir a depressão, especialmente quando o construto da qualidade de vida contempla dimensões como capacidade funcional, aspectos físicos, aspectos emocionais e aspectos sociais, independentemente do perfil de gênero.

2012 Os idosos com ideação suicida mais elevada apresentavam mais depressão. Uma auto percepção de pior saúde e de mais solidão relacionou-se com mais depressão. Uma maior atividade e mais lazer relacionaram-se com menos ideação suicida.

Fonte: Dados da pesquisa, 2018.

A depressão no idoso é um dos transtornos psiquiátricos mais frequentes, embora não seja normal. Acredita-se que conforme o aumento da idade mais sintomas depressivos são apresentados através das queixas intensificadas de doenças e a presença do quadro de ansiedade. Para viver com qualidade a pessoa idosa necessita ter conhecimento sobre sua condição e as doenças que podem the acometer, o que a auxilia na promoção da própria saúde, reduzindo o sofrimento. Contribuindo para o envelhecimento ativo, tornandoa participativa na sociedade, onde demonstra autonomia sobre a própria velhice (DE ANDRADE et al., 2016). 
De acordo com a literatura existem entre 2 a $50 \%$ casos de depressão na população idosa, mas esses dados podem variar de acordo com as delimitações do estudo, como definição de idade, local do estudo e método utilizado para avaliar a depressão. Em geral, pertencer ao sexo feminino, possuir baixo nível socioeconômico, abusar de bebidas alcoólicas, viver sozinho, ter histórico de depressão são fatores de risco que favorecem o aparecimento da depressão (GULLICHI et al., 2016).

O envelhecimento populacional é uma questão mundial. Segundo a OMS 2016, em 2050 cerca de $80 \%$ das pessoas idosas serão procedentes de países emergentes e em desenvolvimento (DE ANDRADE et al., 2016).

A depressão além de constituir-se em um sério problema de saúde, é também considerada um forte fator de risco para outros agravos a saúde, como o uso/abuso de álcool e outras drogas, vindo a desenvolver abuso e dependência. Torna-se oportuno salientar que também existem os casos onde a pessoa começa a usar drogas e a partir daí desenvolve transtorno psiquiátrico, ou em detrimento da predisposição genética, fator comum que pode contribuir para o desenvolvimento destas duas comorbidades (CANTÃO et al., 2015).

As causas para o episódio de depressão estão relacionadas com a perda de um ente querido, a perda da capacidade de aprendizagem além da perda da capacidade de realizar atividades físicas e outros. Já o acolhimento e a prática de atividades sociais, principalmente voluntariada, atividade física e participação em atividade religiosa, são fatores positivos, ou seja, que protegem o aparecimento da depressão (GULLICH et al., 2016).

É importante ressaltar que as informações acerca do diagnóstico, etiologia e características principais da doença devem ser revistas e atualizadas pelos profissionais da saúde, que tem importante papel no cuidado e na atenção a esse público em todos os níveis de saúde. Assim como, possui o compromisso de produzir conhecimento científico que fundamente suas ações e norteiem sua prática (CANTÃo et al., 2015).

É importante que os profissionais empreguem em sua prática ferramentas que promovam suas investigações, como a Escala de Depressão Geriátrica, e que desenvolvam ações de saúde integrais que gerem autonomia e participação do idoso na comunidade, seguindo as diretrizes da Política Nacional da Saúde no ano de 2017, procurando compreender que os fatores sociais, culturais e subjetivos se unem a saúde e qualidade de vida do idoso, acordando para a necessidade da assistência holística (SOUSA et al., 2017).

Um dos fatores decisivos para a adesão ao tratamento é a confiança depositada pelo paciente na prescrição, na equipe de saúde ou no médico. $O$ tratamento usado para tratar essa população inclui a terapia medicamentosa com antidepressivos $(A D)$. É realizado com a administração de medicamentos que pertencem a uma das seguintes classes: antidepressivos tricíclicos (ADTs), inibidores seletivos da recaptação da serotonina (ISRSs), inibidores seletivos da recaptação da serotonina e noradrenalina (ISRSNs), inibidores da monoaminooxidase (iMAOs) e os antidepressivos atípicos (ISTILLI et al., 2010).

Os benzodiazepínicos (BZD) são fármacos com atividade hipnótica e ansiolítica, possuem eficácia nos transtornos ansiosos, além de ação hipnótica, amnéstica, anticonvulsivante e relaxante muscular. Os benzodiazepínicos apresentavam baixos riscos de intoxicações e dependência. Entre os diversos derivados BZD, o diazepam destacou-se com 34 milhões de prescrições no ano de 1991 (CRUZ et al., 2007).

A Fluoxetina antidepressivo da classe dos inibidores seletivos da recaptação da serotonina, é um dos medicamentos mais utilizados no tratamento da depressão, os motivos pelos quais é prescrito incluem: a ansiedade, angústia, insônia, alteração de humor, nervosismo, infelicidade, distimia, distúrbios alimentares, estresse, falta de concentração e desânimo excessivo. (ISTILLI et al., 2010). 
O uso indiscriminado e excessivo desses fármacos pode expor os pacientes a efeitos adversos desnecessários e interações medicamentosas potencialmente perigosas. Idosos, assim consideradas as pessoas com 60 anos de idade ou mais (Ministério da Saúde, 1999), são particularmente vulneráveis aos efeitos adversos de medicamentos, sendo justamente os indivíduos que mais os consomem. Mais de $80 \%$ dos idosos tomam no mínimo um medicamento diariamente, o que aumenta os riscos de uso inapropriado (CRUZ et al., 2009).

Para o uso racional do medicamento, a incorporação do farmacêutico na equipe de saúde é de extrema importância, ressaltando a importância da farmacoepidemiologia, principalmente os estudos de utilização dos medicamentos, que são levantamentos epidemiológicos da utilização de medicamentos utilizados por uma população específica, podendo utilizar ferramentas como a farmacovigilância e farmacoeconomia. Assim, os estudos de utilização dos medicamentos apresentam-se como alternativa para a redução dos custos nos serviços de saúde, pois por meio destes estudos populacionais torna-se possível detectar eventos adversos (LEIRA PEREIRA et al., 2012).

A importância atribuída à ocupação dos tempos livres em idosos: ler, ver televisão, ouvir música, passear, fazer tricô, jogar às cartas, conversar com amigos, sendo que a maior pontuação corresponde maior importância dada à atividade e lazer (DE ALMEIDA et al., 2012).

Godinho at al. (2015), destacam como tratamento alternativo a terapia do riso, que inclui grupo de metodologias e intervenções terapêuticas votadas a realizar experiências de riso que resultem em melhoria das condições de saúde, tanto físicas como emocionais. O riso possui três elementos principais: motor, cognitivo e afetivo; envolvem vários circuitos corticais e subcorticais, sistema límbico, áreas especiais (visual, auditiva e olfativa) e o eixo hipotálamo hipófise, que quando excitados pela ação de rir, liberam endorfinas, encefalinas e neurotransmissores como a serotonina e a dopamina, responsáveis por efeitos psicológicos e físicos associados ao riso (RIPOLL E CASADO, 2010; LANCHEROS et al., 2011). No trabalho de Godinho e seus colaborados foi observado que a terapia do humor foi benéfica para a não progressão da depressão entre os idosos institucionalizados.

A prática do exercício físico cominou proteção para o evento de depressão na população. Existem vários relatos e indicação sobre os efeitos benéficos da atividade física para melhorar a depressão em pessoas idosas. Isso devido ao bem-estar psicológico ocasionado pela influência mútua ou pela formação e acolhimento de redes com relações afetivas estabelecidas. Resultados de um trabalho controlado e randomizado demonstraram que, quanto menor a depressão, o indivíduo aparece mais dispoto a se manter fisicamente ativo e, consequentemente, atenua a possibilidade do regresso dos sintomas depressivos (GULLICH et al., 2016; MENDES et al., 2017). Nesse sentido, Melo at al. (2018) avaliou o efeito da dança de salão no tratamento da depressão, uma vez que produz benefícios físicos, psicológicos e sociais; nos quais os idosos manifestam suas necessidades e debilidades. Neste trabalho foi observado que a prática regular de dança de salão é um instrumento alternativo para o tratamento da depressão em idoso.

\section{CONSIDERAÇÕES FINAIS}

Com base nos resultados, conclui-se que a necessidade de agir de modo preventivo, junto dos idosos, é premente. A prática e o desenvolvimento de atividades de lazer têm-se mostrado na vida dos idosos como um fator de grande importância. O lazer contribui para um melhor estado de espírito e no caso dos mais idosos, diminui os efeitos decorrentes do processo de envelhecimento. A prática de atividade física atribuiu proteção para o aparecimento da depressão na população, o indivíduo manifestar-se mais predisposto a se manter fisicamente ativo e, com isso, reduz a perspectiva de retorno dos sintomas depressivos.

O subdiagnóstico da doença tem um impacto importante nas relações interpessoais do idoso e no controle de outras doenças. É importante que os familiares e amigos estejam atentos aos sintomas que possam surgir, 
devendo recorrer a um profissional de saúde para esclarecimento de dúvidas. A família durante a terceira idade pode promover inúmeros benefícios à saúde, como evitar a depressão, os problemas do coração e muito mais. Mais do que triste, a solidão é perigosa para a saúde dos idosos, é tão prejudicial ao corpo quanto à obesidade na terceira idade. Pode existir uma maior sensibilidade à dor, predisposição à infecção e maior descontentamento porque a solidão é um exacerbador dessas condições, tornando-as mais evidentes.

\section{REFERÊNCIAS}

1. ALMEIDA L, SÓNIA Q. "Depressão e ideação suicida em idosos institucionalizados e não institucionalizados em Portugal." Acta Medica Portuguesa, 2012: 25(6): 350-358.

2. BRASIL. IBGE. Censo Demográfico, 2010. Disponível em www.ibge.gov.br

3. CANTÃO $L$ et al. Perfil sociodemográfico e clínico de idosos com depressão e o uso de substâncias psicoativas. Revista da Rede de Enfermagem do Nordeste, 2015; 16 (3)

4. CIASCA EC. Arteterapia e depressão: efeitos da arteterapia como terapia complementar no tratamento da depressão em idosos. Diss. Universidade de São Paulo, 2017; 345 p.

5. COHEN R, PASKULIN LMG, PRIEB RGG. Prevalência de sintomas depressivos entre idosos em um serviço de emergência. Revista Brasileira de Geriatria e Gerontologia, 2015; 18 (2): 307-317.

6. CRUZ AV. et al. Uso crônico de diazepam em idosos atendidos na rede pública em Tatuí-SP. Revista de Ciências Farmacêuticas Básica e Aplicada,2009; 27(3): 259-267.

7. DE ANDRADE ABCA, FERREIRA AA, DE AGUIAR MJG. CONHECIMENTO DOS IDOSOS SOBRE OS SINAIS E SINTOMAS DA DEPRESSÃO. Saúde em Redes, 2016; 2(2): 157-166.

8. GARCÍA EAL. José Fernando Tovar Valle, and Camilo Augusto Rojas Bermúdez. "Risa y salud: abordajes terapéuticos." MedUNAB, 2011; 14(1): 69-75.

9. GODINHO BPW, et al. "Efeito da intervenção clown no padrão de depressão de idosos em instituição de longa permanência." Ciencia y Enfermería, 2015; 21(2): 765-771.

10. GULLICH I, DURO SMS, CESAR JA. Depressão entre idosos: um estudo de base populacional no Sul do Brasil. Revista Brasileira de Epidemiologia, 2016; 19(1): 691-701.

11. ISTILLI PT, et al. Antidepressivos: uso e conhecimento entre estudantes de enfermagem. Revista LatinoAmericana de Enfermagem, 2010; 18 (3): 132-139.

12. LEAL LN. "População idosa vai triplicar entre 2010 e 2050, aponta publicação do IBGE." O Estado de S. Paulo (2016).

13. LEIRA PEREIRA LR, FREITAS O, QUEIROZ NETTO MU. Antidepressivos e Benzodiazepínicos: estudo sobre o uso racional entre usuários do SUS em Ribeirão Preto-SP. Revista de Ciências Farmacêuticas Básica e Aplicada, 2012;33 (1):77-81.

14. LIMA AMP, et al. Depressão em idosos: uma revisão sistemática da literatura. Revista de Epidemiologia e Controle de Infecção, 2016; 6(2): 96-103.

15. MELO CC, et al. "A Influência doTempo de Prática de Dança de Salão nos Níveis de Depressão de Idosos." Revista de psicología del deporte, 2018; 27(4): 0067-73.

16. MENDES, G AB, et al. "RELAÇÃO ENTRE ATIVIDADE FísICA E DEPRESSÃO EM IDOSOS: UMA REVISÃO INTEGRATIVA." Revista de Atenção à Saúde (antiga Rev. Bras. Ciên. Saúde, 2017; 15(53): 110116.

17. MIGUEL MAL. A dor crônica no idoso e seu impacto no desenvolvimento da depressão. 2016.

18. MIRANDA, LCV, SOARES SM, SILVA PAB. Qualidade de vida e fatores associados em idosos de um Centro de Referência à Pessoa Idosa. Ciência \& Saúde Coletiva, 2016; 21(2): 3533-3544.

19. MORAES $\mathrm{H}$, et al. O exercício físico no tratamento da depressão em idosos: revisão sistemática. Rev Psiquiatr Rio Gd Sul, 2007; 29 (1): 70-9.

20. NÓBREGA IRAP, et al. Fatores associados à depressão em idosos institucionalizados: revisão integrativa. Saúde em Debate, 2015; 39(3): 536-550.

21. RAMOS GCF et al. "Prevalência de sintomas depressivos e fatores associados em idosos no norte de Minas Gerais: um estudo de base populacional." J Bras Psiquiatr, 2015; 64 (2): 122-131.

22. RIPOLL RM, ISABEL QC. "Laughter and positive therapies: modern approach and practical use in medicine." Revista de Psiquiatría y Salud Mental (English Edition), 2010; 3 (1): 27-34.

23. RUFINE MC, et al. Perfil psicológico de gênero, qualidade de vida e depressão: proposta de um modelo causal em mulheres idosas. Psicologia Argumento, 2017; 32(1): 31-41.

24. SOUSA KA, et al. Prevalência de sintomas de depressão em idosos assistidos pela estratégia de saúde da família. REME rev. min. enferm, 2017; 21(2):.82-93.

25. SOUZA MT, SILVA MD, CARVALHO R. Revisão integrativa: o que é e como fazer. Einstein [Internet]. 2010; $8(1): 102-6$. 